\title{
Curva dos valores normais de peso fetal estimado por ultra-sonografia segundo a idade gestacional
}

\author{
Curve of normal estimated fetal weight values \\ by ultrasound according to gestation age
}

José Guilherme Cecatti 1

Maria Regina Marrocos Machado 1

Fernanda Fioravanti Azank dos Santos 1

Emílio Francisco Marussi 1

1 Centro de Atenção Integral à Saúde da Mulher, Departamento de Tocoginecologia Faculdade de Ciências Médicas, Universidade Estadual de Campinas. Rua Alexander Fleming 101, Campinas, $S P$

13081-970, Brasil. cecatti@unicamp.br

\begin{abstract}
Objective: to evaluate the evolution of estimated fetal weight in normal pregnancies in Campinas, Brazil, between 20 to 42 weeks. Design and methods: A descriptive study was performed, including 2,874 normal pregnant women studied through routine obstetric ultrasound with fetal biometry, using the Hadlock (1991) formula for fetal weight estimation. Data were evaluated by fitting a curve with the 10th, 50th, and 90th percentiles of fetal weight for gestational age, subsequently submitted to a smoothing procedure by quadratic polynomial adjustment. Results: the 50th percentile was $368 \mathrm{~g}$ at 20th week, 1,512g at 30th, and 3,417g at 42nd week. There was a mean weight increase of $200 \mathrm{~g}$ per week from the 27 th to 38 th week, when the gain decreased. Conclusions: The availability of a Brazilian curve of normal estimated fetal weight will allow for prenatal ultrasound diagnosis of deviations.
\end{abstract}

Key words Fetal Weight; Gestational Age; Fetal Growth Retardation; Prenatal Ultrasonography

Resumo Este trabalho teve por objetivo avaliar a evolução do peso fetal estimado em gestações normais de 20 a 42 semanas. Quanto ao desenho e métodos empregados na pesquisa, realizou-se estudo descritivo de um universo constituído por 2.874 gestantes normais da cidade de Campinas, efetuando-se exame ultra-sonográfico obstétrico de rotina com medida da biometria fetal e utilizando-se, para o cálculo do peso fetal, a fórmula de Hadlock et al. (1991). Calcularam-se os valores dos percentis 10, 50 e 90 do peso fetal, por idade gestacional, com construção de curva posteriormente alisada mediante ajuste polinomial de 2 o grau. Como resultado, obteve-se que o valor do percentil 50 foi de 368 gramas na 20 a semana, 1.512 gramas na 30 a semana, atingindo 3.417 gramas na 42 a semana. Constatou-se ter havido ganho médio de 200 gramas de peso por semana, de 27 a 38 semanas, a partir de quando o incremento diminuiu. Concluiu-se que a disponibilidade de curva brasileira do peso fetal estimado normal permitirá o diagnóstico pré-natal de seus desvios pela ultra-sonografia.

Palavras-chave Peso Fetal; Idade Gestacional; Retardo do Crescimento Fetal; Ultrasonografia Pré-Natal 


\section{Introdução}

A determinação do peso fetal estimado (PFE) pela ultra-sonografia é importante meio de verificar o bem-estar fetal e de avaliar a evolução de seu crescimento no decorrer da gestação, assim como reduzir a morbidade e mortalidade associadas ao retardo de crescimento intraútero (RCIU) (Hadlock et al., 1991).

Várias fórmulas foram criadas para estimar o peso fetal a partir de medidas da biometria, podendo incluir o diâmetro biparietal, a medida do fêmur e a circunferência abdominal. Os trabalhos de Hadlock et al. (1983) mostram ser necessárias, pelo menos, três medidas fetais, que são: o diâmetro biparietal ou a circunferência cefálica, como índice de crescimento da cabeça; circunferência abdominal, como índice de crescimento do corpo; e, por fim, o comprimento do fêmur, como índice de crescimento em estatura.

Há múltiplos determinantes não-patológicos do peso neonatal ao nascimento. No entanto, existem divergências entre os padrões adotados como normais, incluindo-se aí variações das características populacionais, como, por exemplo, fatores antropométricos e demográficos maternos, fatores ambientais, tamanho amostral adotado em estudos preliminares, bem como critérios de exclusão e fórmulas matemáticas utilizadas para cálculo (Goldenberg et al., 1989).

Dentre estes fatores variantes, o mais importante deles é o potencial intrínseco de crescimento individual do feto (Ott, 1990). É possível encontrar estudos comparativos de populações brancas e negras/hispânicas em que não se verificou diferença significativa entre os parâmetros utilizados para o cálculo do peso fetal (diâmetro biparietal, circunferência cefálica, circunferência abdominal e comprimento femural) (Hadlock et al., 1990).

Outros fatores podem ainda influenciar e modificar valores de referência adotados para a estimativa do peso fetal. No que se refere ao tipo de população estudada, por exemplo, é preciso tomar em conta se é proveniente de centros de referência hospitalares ou da população geral, da mesma forma que a raça, estatura e ossatura maternas, a divisão em curvas diferentes por sexo fetal, além da determinação da idade gestacional - mesmo que o seja por ultra-som precoce ou pela data da última menstruação (DUM) de certeza - se o ajustamento de número de dias se faz para mais ou menos no número de semanas.

Quanto à técnica para a estimativa do peso fetal, esta pode sofrer variações que vão desde várias fórmulas de diferentes autores - já inclusas nos aparelhos de ultra-sonografia - até variações intra e inter-observador. Em estudos realizados por Chang et al. (1993), o desvio-padrão intra-observador foi menor que 75g. $\mathrm{O}$ intervalo de confiança a $95 \%$ da variação interobservador foi de $-187,3 \mathrm{a}+139,8 \mathrm{~g}$, usando uma única fórmula, e de $-159,9 \mathrm{a}+124,3 \mathrm{~g}$ quando duas fórmulas foram utilizadas. Assim, os resultados da estimativa de peso fetal por ultrasom foram considerados reprodutíveis.

A presença de patologias maternas, bem como as fetais, também deve ser conhecida para que se possa avaliar sua possível influência no crescimento fetal. Características fisiológicas maternas - como peso e altura maternos e paridade - podem igualmente influenciar o ganho de peso fetal, em especial, no 30 trimestre da gestação (De Jong et al., 1998).

É fundamental o discernimento entre o baixo peso ao nascimento como conseqüência de retardo de crescimento intra-uterino e aquele resultante de baixo potencial de crescimento inerente, para que se possa entender e manejar clinicamente o retardo de crescimento intraútero. Há diferença entre tamanho e crescimento, a qual, até há poucos anos atrás, não havia sido elucidada. O diagnóstico de retardo de crescimento intra-útero pode ser dado somente se existir desvio significativo dos padrões normais de crescimento, o que requer, no mínimo, duas medidas ultra-sonográficas da biometria fetal (Owen \& Ogston, 1998).

Além disso, é preciso que se esteja atento à diferença entre o RCIU simétrico e o assimétrico. Suas causas estão relacionadas respectivamente a processos patológicos crônicos e agudos. Na primeira condição, todas as medidas da biometria fetal encontram-se a, pelo menos, dois desvios-padrões abaixo da média. Já na segunda condição, há diminuição da circunferência abdominal fetal em relação ao crescimento do fêmur. Esta diferenciação é importante para que se intervenha no processo causal. Com relação ao peso, para que um feto seja considerado com retardo de crescimento intra-útero, ele deve estar com peso abaixo do percentil $10 \mathrm{da}$ curva de peso para a idade gestacional e/ou apresentar relação fêmur/circunferência abdominal menor que $24 \%$.

O aumento do peso fetal com diagnóstico de macrossomia acarreta desde mudança na conduta obstétrica até a necessidade de investigação mais apurada da glicemia materna e do recém-nascido. Já o diagnóstico de feto com retardo de crescimento intra-útero pode significar, tanto para o obstetra como para a mulher, situação de risco, o que, muitas vezes, não exis- 
te. Comparando-se o crescimento fetal de nossa população com o de populações que apresentam diferentes características sócio-econômicas, demográficas e antropométricas, é possível superdiagnosticar situações de retardo do crescimento intra-útero.

A identificação correta de desvios do crescimento fetal é imprescindível, o que implica melhor assistência materna, maior número de retornos à consulta médica e, inclusive, a realização de ecografias seriadas de controle.

Nos países em desenvolvimento, o peso ao nascimento é provavelmente o fator mais importante, influenciando a morbidade e mortalidade neonatal, além de exercer papel fundamental na determinação da mortalidade pósneonatal. Tal fato fica evidente ao verificar-se que o risco de morte neonatal é quatro vezes menor nas crianças que nascem com peso de 2.500-2.999g, e dez vezes menor naquelas com 3.000-3.499g (Ashworth, 1998).

Assim, em se tratando de problema de saúde pública, estudos clínicos e epidemiológicos têm direcionado a atenção aos fatores causais que podem influenciar o peso fetal (Kramer, 1987). A hipótese mais aceita é de que a ocorrência de baixo peso ao nascimento seja multifatorial. No entanto, dois fatores básicos devem ser diferenciados no diagnóstico de baixo peso ao nascimento: a prematuridade (gestação de menor duração) e o retardo de crescimento intra-útero, com a possibilidade da presença de ambos.

Desta forma, as causas de baixo peso ao nascimento são mais relacionadas, em países desenvolvidos, à prematuridade, em contraposição àqueles em desenvolvimento, cujas causas estão mais relacionadas ao retardo de crescimento intra-útero.

Fatores como desnutrição materna, idade materna avançada, antecedentes de filhos com baixo peso, história de partos prematuros, multiparidade, a falta de acompanhamento prénatal e outros agiriam, isolada e associadamente, com maior intensidade em nossa população em comparação a outros países, cujas curvas de peso fetal são adotadas no Brasil para determinar o peso fetal ao nascimento. No entanto, o primeiro passo para comparação mais precisa e correta do peso fetal estimado em relação à idade gestacional é a determinação precisa desta.

Assim, foi objetivo deste estudo determinar os valores dos percentis 10, 50 e 90 do peso fetal estimado, de 20 a 42 semanas, em gestantes normais, para a população de Campinas.

\section{Sujeitos e métodos}

O presente estudo foi descritivo da elaboração de curva de valores normais do peso fetal estimado. Foram estudadas 2.874 gestantes normais, de 20 a 42 semanas, da cidade de Campinas, estado de São Paulo, no período de agosto de 1997 a abril de 1998, identificando-se as gestantes que poderiam ser incluídas no estudo a partir de exames de rotina feitos pelo serviço (Perrotti, 1998).

Foram incluídas as gestantes com data do primeiro dia do último período menstrual normal - quando conhecido com segurança -, expressando-se em semanas ou dias completos a idade gestacional (Araújo \& Maretti, 1991); caso esta primeira condição não fosse cumprida, a idade gestacional foi estimada por exame ultra-sonográfico pélvico precoce, efetuado antes de 24 semanas, adotando-se valor único da mesma quando da disponibilidade de ambos. Para que a idade gestacional de 20 a 42 semanas fosse considerada confiável, verificou-se a ausência de uso de anticoncepcionais orais nos últimos três meses ou injetáveis nos últimos seis meses anteriores a essa gestação.

Não foram incluídas as gestantes que apresentassem quaisquer das seguintes condições: hipertensão arterial crônica; hipertensão gestacional; diabetes mellitus; rotura de membranas; senescência placentária precoce (segundo classificação de Grannum et al., 1979); gestação múltipla; anomalia congênita fetal; óbito fetal; isoimunização fetal; afora outras situações, tais como: doenças metabólicas, nefropatias, cardiopatias, hiper e hipotireoidismo materno. Tais critérios de exclusão foram investigados, em cada caso, através de check-list aplicado antes da admissão do caso ao estudo.

A variável dependente do estudo foi o peso fetal estimado, determinado pelo exame ultrasonográfico, para aquele momento presente, pela fórmula de Hadlock et al. (1991), com erro estimado em 10\%, expresso em gramas exatas: Equação $\left[\log _{10}\right.$ (Peso ao nascimento) $]=1,3596$ 0,00386 (CA) $(\mathrm{F})+0,0064$. (CC) + 0,00061 (DBP) $(\mathrm{CA})+0,0424(\mathrm{CA})+0,174(\mathrm{~F})$

CA: circunferência abdominal

CC: circunferência cefálica

$F$ : comprimento do fêmur

DBP: diâmetro biparietal

Para efetuar os exames ultra-sonográficos nas gestantes, foram utilizados os aparelhos Toshiba SSA-140 e Acuson XP4A, usando-se sonda convexa de 3,75 mHZ em ambos, normalmente adotada como padrão para exames ultra-sonográficos obstétricos, sendo realizados por um único observador. 
Inicialmente, construiu-se uma curva de valores normais do peso fetal estimado para cada idade gestacional - no período de 20 a 42 semanas de gestação - através da estimativas dos percentis 10, 50 e 90. Posteriormente, esta curva foi submetida a alisamento por procedimento de ajuste polinomial de 2 o grau (Armitage, 1974).

\section{Resultados}

A população estudada foi composta de $70 \%$ de mulheres brancas e de $66 \%$ com baixo nível de escolaridade e, indiretamente, de nível sócioeconômico (Perrotti, 1998).

A média do peso fetal estimado por semana de gestação está na Tabela 1. A curva de valores normais com os percentis 10,50 e 90 está na Tabela 2, expressa graficamente na Figura 1. A curva alisada é exposta na Tabela 3 e na Figura 2. Os valores percentilares não alisados (anteriores ao ajuste polinomial) mostram estabilização do peso fetal a partir da 39a semana.

Tabela 1

Média e desvio-padrão do peso fetal estimado de gestantes normais, segundo a idade gestacional. Campinas, 1998.

\begin{tabular}{|c|c|c|c|}
\hline \multirow{2}{*}{$\begin{array}{l}\text { Idade gestacional } \\
\text { (semanas) }\end{array}$} & \multicolumn{2}{|c|}{ Peso fetal estimado (g) } & \multirow[t]{2}{*}{$\mathrm{n}$} \\
\hline & $\bar{x}$ & DP & \\
\hline 20 & 368,84 & 45,78 & 124 \\
\hline 21 & 427,05 & 46,49 & 129 \\
\hline 22 & 504,05 & 62,14 & 123 \\
\hline 23 & 581,79 & 59,97 & 122 \\
\hline 24 & 677,95 & 62,76 & 124 \\
\hline 25 & 776,53 & 84,66 & 120 \\
\hline 26 & 908,45 & 112,90 & 120 \\
\hline 27 & $1.045,82$ & 112,70 & 120 \\
\hline 28 & $1.190,82$ & 127,95 & 120 \\
\hline 29 & $1.360,74$ & 152,82 & 120 \\
\hline 30 & $1.527,48$ & 210,20 & 122 \\
\hline 31 & $1.715,41$ & 194,27 & 123 \\
\hline 32 & $1.926,81$ & 200,02 & 128 \\
\hline 33 & $2.101,73$ & 201,63 & 130 \\
\hline 34 & $2.294,31$ & 193,35 & 132 \\
\hline 35 & $2.536,17$ & 225,47 & 136 \\
\hline 36 & $2.725,14$ & 237,23 & 140 \\
\hline 37 & $2.943,85$ & 305,21 & 132 \\
\hline 38 & $3.119,97$ & 335,67 & 122 \\
\hline 39 & $3.267,18$ & 372,36 & 121 \\
\hline 40 & $3.344,06$ & 383,83 & 123 \\
\hline 41 & $3.373,33$ & 396,12 & 122 \\
\hline 42 & $3.417,18$ & 378,43 & 121 \\
\hline
\end{tabular}

O peso fetal estimado variou conforme o esperado, com ganho médio de $200 \mathrm{~g}$ de peso por semana da gestação a partir da $27 \underline{\text { a }}$ até a $38 \underline{\text { a }}$ semana, com incremento menor das 39 às 42 semanas. $\mathrm{O}$ percentil 50 apresentou-se em torno de $368 \mathrm{~g}$ na 20 a semana, atingindo $1.000 \mathrm{~g}$ na 27 a semana, $2.000 \mathrm{~g}$ na 33 a semana e $3.000 \mathrm{~g}$ na 37 a semana.

\section{Discussão}

Apesar das diferenças étnicas e econômicas das populações, houve concordância da curva construída em relação à curva de Hadlock et al. (1991); entretanto, foram constatados valores discretamente inferiores na curva construída para as idades gestacionais maiores que 30 semanas. Os valores do percentil 90 da curva construída aproximaram-se dos valores do percentil 50 de Hadlock et al. (1991). Isto pode mostrar que existe tendência para que o peso fetal ao nascimento seja menor em todos os percentis e nas idades gestacionais após 30 semanas, quando comparado à curva de Hadlock et al. (1991).

A população estudada apresentou distribuição pelo nível de escolaridade e, portanto, indiretamente, do nível sócio-econômico que reflete a realidade brasileira, com $66 \%$ da população proveniente de nível mais baixo e $34 \%$ correspondendo a nível mais elevado (Perrotti, 1998).

Embora crianças negras pesem, em geral, menos que as brancas, e seu 10o percentil seja mais baixo, não está claro se esta diferença deve-se somente a menor potencial genético para crescimento nas crianças negras. No entanto, entre as crianças negras há mais fatores de risco para baixo peso ao nascimento, incluindo condições sócio-econômicas mais baixas e maior ocorrência de hipertensão materna. Estudos americanos sobre RCIU destacam ser melhor o uso de populações heterogêneas quanto à raça (Goldenberg et al., 1989). No presente estudo, não se fez separação entre população branca e não branca, pois houve distribuição equivalente à da população brasileira, com $70 \%$ de branca e $30 \%$ de não branca, favorecendo sua aplicabilidade clínica (Perrotti, 1998).

Fetos masculinos comumente pesam mais que os femininos, além de os valores do percentil 10 serem maiores nos masculinos, pelo menos em algumas populações. Além disso, não há maior risco na gestação em relação ao sexo fetal (excluindo patologias ligadas ao sexo) (Goldenberg et al., 1989). Portanto, não se separaram os percentis de peso fetal pelo sexo, 
Tabela 2

Valores dos percentis 10, 50 e 90 do peso fetal estimado de gestantes normais, segundo a idade gestacional.

Campinas, 1998.

\begin{tabular}{|c|c|c|c|c|}
\hline $\begin{array}{l}\text { Idade gestacional } \\
\text { (semanas) }\end{array}$ & \multicolumn{3}{|c|}{ Peso fetal estimado (g) } & $n$ \\
\hline 20 & 316,0 & 368,5 & 424,0 & 124 \\
\hline 21 & 367,0 & 425,0 & 489,0 & 129 \\
\hline 22 & 432,0 & 505,0 & 570,0 & 123 \\
\hline 23 & 510,0 & 578,0 & 658,0 & 122 \\
\hline 24 & 595,0 & 684,5 & 747,0 & 124 \\
\hline 25 & 672,5 & 774,5 & 864,5 & 120 \\
\hline 26 & 783,0 & 917,5 & $1.048,0$ & 120 \\
\hline 27 & 902,0 & $1.043,5$ & $1.189,0$ & 120 \\
\hline 28 & $1.041,5$ & $1.170,0$ & $1.376,5$ & 120 \\
\hline 29 & $1.200,5$ & $1.341,5$ & $1.550,0$ & 120 \\
\hline 30 & $1.337,0$ & $1.512,5$ & $1.733,0$ & 122 \\
\hline 31 & $1.518,0$ & $1.700,0$ & $1.975,0$ & 123 \\
\hline 32 & $1.737,0$ & $1.908,5$ & $2.154,0$ & 128 \\
\hline 33 & $1.856,0$ & $2.101,5$ & $2.343,0$ & 130 \\
\hline 34 & $2.070,0$ & $2.288,5$ & $2.535,0$ & 132 \\
\hline 35 & $2.256,0$ & $2.527,0$ & $2.829,0$ & 136 \\
\hline 36 & $2.390,0$ & $2.755,5$ & $3.006,0$ & 140 \\
\hline 37 & $2.583,0$ & $2.952,5$ & $3.307,0$ & 132 \\
\hline 38 & $2.704,0$ & $3.127,5$ & $3.491,0$ & 122 \\
\hline 39 & $2.816,0$ & $3.305,0$ & $3.772,5$ & 121 \\
\hline 40 & $2.826,0$ & $3.334,0$ & $3.870,0$ & 123 \\
\hline 41 & $2.844,0$ & $3.411,0$ & $3.823,5$ & 122 \\
\hline 42 & $2.938,0$ & $3.385,5$ & $3.865,5$ & 121 \\
\hline
\end{tabular}

Figura 1

Valores dos percentis 10, 50 e 90 do peso fetal estimado de gestantes normais, segundo a idade gestacional. Campinas, 1998.

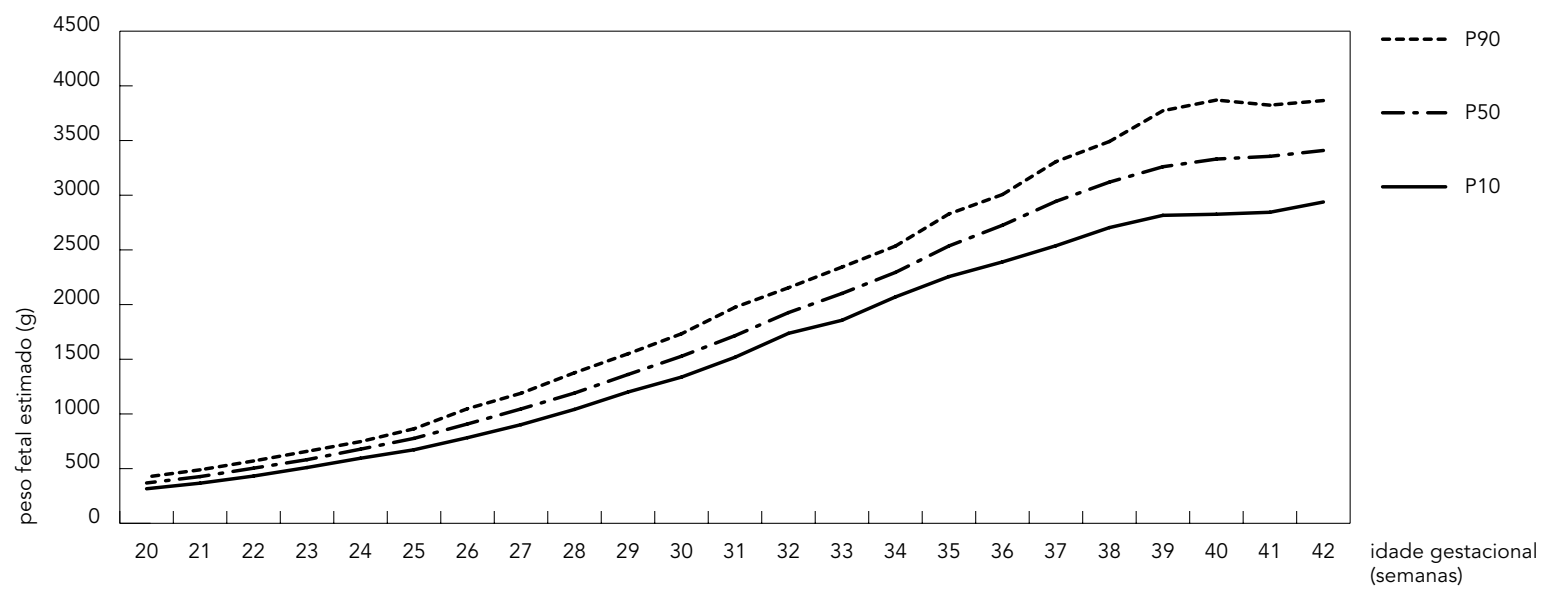

* Peso fetal estimado pela fórmula de Hadlock et al. (1991). 
Valores estimados* para os percentis 10,50 e 90 do peso fetal estimado de gestações normais segundo a idade gestacional. Campinas, 1998.

\begin{tabular}{|c|c|c|c|c|}
\hline $\begin{array}{l}\text { Idade gestacional } \\
\text { (semanas) }\end{array}$ & percentil 10 & $\begin{array}{l}\text { fetal estimad } \\
\text { percentil } 50\end{array}$ & percentil 90 & $n$ \\
\hline 20 & 95,5 & 284,2 & 324,2 & 124 \\
\hline 21 & 231,7 & 389,1 & 442,5 & 129 \\
\hline 22 & 367,9 & 499,0 & 566,6 & 123 \\
\hline 23 & 504,1 & 614,1 & 696,5 & 122 \\
\hline 24 & 640,2 & 734,3 & 832,1 & 124 \\
\hline 25 & 776,4 & 859,6 & 973,5 & 120 \\
\hline 26 & 912,6 & 990,0 & $1.120,7$ & 120 \\
\hline 27 & $1.048,8$ & $1.125,5$ & $1.273,7$ & 120 \\
\hline 28 & $1.184,9$ & $1.266,1$ & $1.432,4$ & 120 \\
\hline 29 & $1.321,1$ & $1.411,9$ & $1.596,9$ & 120 \\
\hline 30 & $1.457,3$ & $1.562,7$ & $1.767,2$ & 122 \\
\hline 31 & $1.593,5$ & $1.718,7$ & $1.943,2$ & 123 \\
\hline 32 & $1.729,6$ & $1.879,8$ & $2.125,0$ & 128 \\
\hline 33 & $1.865,8$ & $2.046,0$ & $2.312,6$ & 130 \\
\hline 34 & $2.002,0$ & $2.217,3$ & $2.506,0$ & 132 \\
\hline 35 & $2.138,2$ & $2.393,8$ & $2.705,1$ & 136 \\
\hline 36 & $2.274,3$ & $2.575,3$ & $2.910,0$ & 140 \\
\hline 37 & $2.410,5$ & $2.762,0$ & $3.120,7$ & 132 \\
\hline 38 & $2.546,7$ & $2.953,7$ & $3.337,2$ & 122 \\
\hline 39 & $2.682,9$ & $3.150,6$ & $3.559,4$ & 121 \\
\hline 40 & $2.819,0$ & $3.352,6$ & $3.787,4$ & 123 \\
\hline 41 & $2.955,2$ & $3.559,7$ & $4.021,1$ & 122 \\
\hline 42 & $3.091,4$ & $3.772,0$ & $4.260,7$ & 121 \\
\hline
\end{tabular}

* Ajustes polinomiais de 1ㅇ e 2o graus:

Peso fetal estimado $=-1607,72+(59,22 \times I G)+(1,60 \times 1 G 2) \&$ valor estimado $<0$

como algumas curvas preconizam, pois pretendeu-se demonstrar a evolução do peso de maneira global, ou seja, mesmo nas situações em que não se conhece o sexo fetal. Curvas de peso fetal diferentes em relação ao sexo fetal já foram construídas, mas esta diferença não se mostrou estatisticamente significativa (Ott et al., 1985; Secher et al., 1987).

Quando se dispõe de valores de peso fetal estimado de gestantes normais, ao longo da gestação é possível diagnosticar desvio da normalidade pela presença de RCIU em um ponto isolado de observação ou em curva de crescimento ao longo do tempo. Para isso basta a realização de exame de ultra-som por intermédio de equipamento adequado e profissional com capacitação técnica para medidas de biometria fetal.

Os resultados mostraram-se bastante similares em relação a outras curvas existentes na literatura para populações tanto étnica quanto economicamente diferentes, porém com valo- res discretamente inferiores a partir da 30a semana. Talvez esta diminuição do peso fetal no terceiro trimestre, ocasião em que o feto deveria ganhar mais peso, possa direcionar para intensificação nos cuidados pré-natais neste período, desde a alimentação materna até a busca e controle das patologias maternas mais freqüentes próximas ao termo.

Utilizando curva efetuada com dados de uma população brasileira, estar-se-á mais próximo à realidade sócio-econômica e às características demográficas maternas de cada caso a ser avaliado, ou seja, aos fatores causais de baixo peso ao nascimento mais prevalentes no país. Com isso, pode-se supor que os resultados devam ser melhor avaliados, facilitando a interpretação dos mesmos para possível melhoria no atendimento da saúde materna e neonatal. Deve-se ainda considerar que o exame de ultrasonografia é realidade e que grande parcela das mulheres grávidas a ele recorrem em algum momento da gestação, motivo pelo qual isso 
Valores estimados* para os percentis 10,50 e 90 do peso fetal estimado de gestantes normais, segundo a idade gestacional. Campinas, 1998.

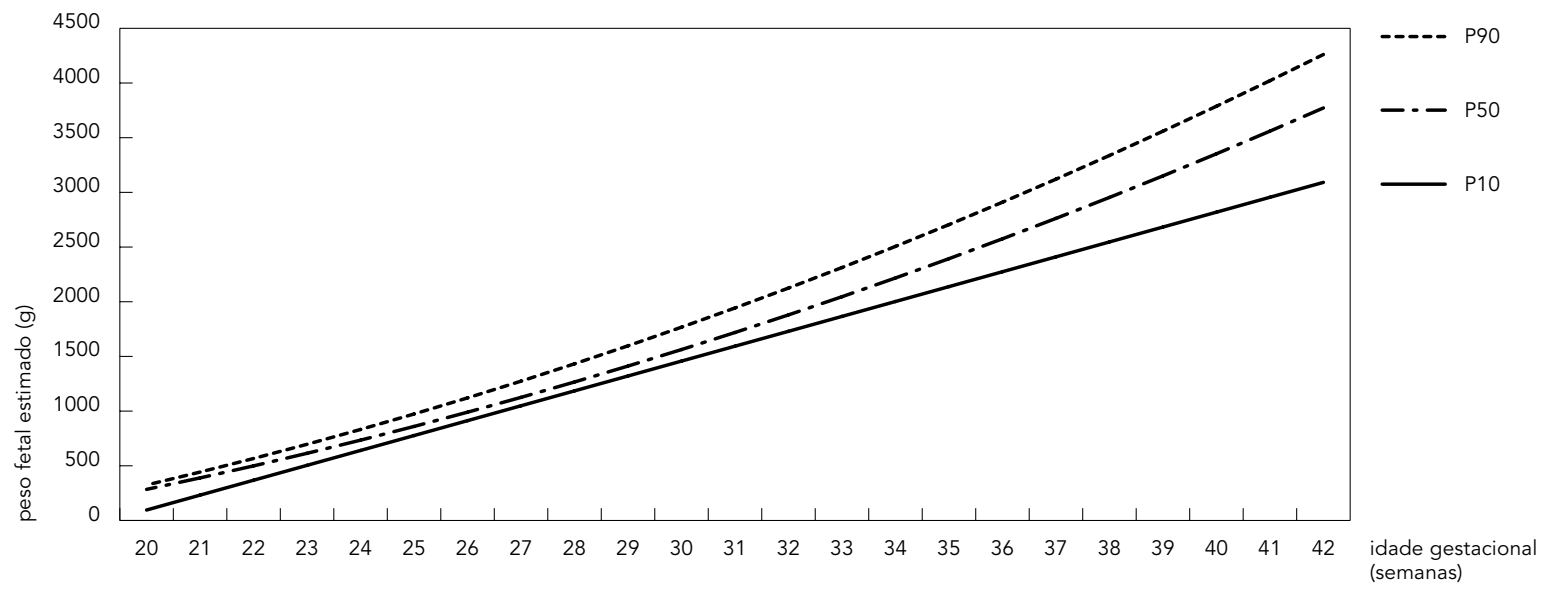

* Ajustes polinomiais de 1 o e 2 o graus

não representaria gasto excedente de grande monta na assistência à mulher grávida.

Finalmente resta ainda uma grande dúvida a ser respondida. Qual a melhor curva de peso fetal estimado a ser utilizada como referência para uma população de gestantes brasileiras? Qual a variabilidade entre a estimativa do peso fetal por ultra-sonografia e o peso real medido ao nascimento? Estas perguntas remetem à necessidade de dar continuidade ao estudo da questão, provavelmente mediante pesquisas que avaliem esta variabilidade e também o desempenho destas curvas, tanto a bruta quanto a alisada, de PFE no diagnóstico de situações de RCIU e recém-nascidos PIG (pequeno para idade gestacional).

\section{Referências}

ARAÚJO, J. O. \& MARETTI, M., 1991. Anamnese e exame físico. In: Obstetrícia (J. Rezende, ed.), pp. 142152, 6a Ed., Rio de Janeiro: Editora Guanabara Koogan.

ARMITAGE, P., 1974. Statistical Methods in Medical Research. New York: John Wiley \& Sons.

ASHWORTH, A., 1998. Effects of intrauterine growth retardation on mortality and morbidity in infants and young children. European Journal of Clinical Nutrition, 52(Sup. 1):S34-S41.

CHANG, T. C.; ROBSON, S. C.; SPENCER, J. A. \& GALLIVAN, S., 1993. Ultrasonic fetal weight estimation: Analysis of inter- and intra-observer variability. Journal of Clinical Ultrasound, 21:515-519.

DE JONG, C. L.; GARDOSI, J.; BALDWIN, C.; FRANCIS, A.; DEKKER, G. A. \& VAN GEIJN, H. P., 1998. Fetal weight gain in a serially scanned high-risk population. Ultrasound in Obstetrics and Gynecology, 11:39-43. 
GOLDENBERG, R. L.; CUTTER, G. R.; HOFFMAN, H. J.; FOSTER, J. M.; NELSON, K. G. \& HAUTH, J. C., 1989. Intrauterine growth retardation: Standards for diagnosis. American Journal of Obstetrics and Gynecology, 161:271-277.

GRANNUM, P. A. T.; BERKOWITZ, R. L. \& HOBBINS, J. C., 1979. The ultrasonic changes in the maturing placenta and their relationship to fetal pulmonic maturity. American Journal of Obstetrics and Gynecology, 133:915-922.

HADLOCK, F. P.; HARRIST, R. B. \& MARTINEZ-POYER, J., 1991. In utero analysis of fetal growth: A sonographic weight standard. Radiology, 181:129-133.

HADLOCK, F. P.; HARRIST, R. B.; SHAH, Y. P.; SHARMAN, R. S. \& PARK, S. K., 1990. Sonographic fetal growth standards. Are current data applicable to a racially mixed population? Journal of Ultrasound in Medicine, 9:157-160.

HADLOCK, F. P.; HARRIST, R. B.; SHARMAN, R. S.; DETER, R. L. \& SEUNG, K. P., 1983. Estimation of fetal weight with the use of head, body, and femur measurements - A prospective study. American Journal of Obstetrics and Gynecology, 151:333-337.
KRAMER, M. S., 1987. Intrauterine growth and gestational duration determinants. Pediatrics, 80:502511.

OTT, W. J., 1990. Defining altered fetal growth by second-trimester sonography. Obstetrics and Gynecology, 75:1053-1059.

OTT, W. J.; DOYLE, S. \& FLAMM, S., 1985. Accurate ultrasonic estimation of fetal weight. American Journal of Perinatology, 2:178-182.

OWEN, P. \& OGSTON, S., 1998. Conditional centiles for the quantification of fetal growth. Ultrasound in Obstetrics and Gynecology, 11:110-117.

PERROTTI, M. R. M., 1998. Curva dos Valores de Índice de Líquido Amniótico em Gestantes Normais. Dissertação de Mestrado, Campinas: Faculdade de Ciências Médicas, Universidade Estadual de Campinas.

SECHER, N. J.; KERN-HANSEN, P.; THOMSEN, B. L. \& KEIDING, N., 1987. Growth retardation in preterm infants. British Journal of Obstetrics and Gynaecology, 94:115-120. 